\title{
Near-field resonance at far-field-induced transparency in diffractive arrays of plasmonic nanorods
}

\author{
S. R. K. Rodriguez, ${ }^{1, *}$ O. T. A. Janssen, ${ }^{2}$ G. Lozano, ${ }^{1}$ A. Omari, ${ }^{3,4}$ Z. Hens, ${ }^{3,4}$ and J. Gómez Rivas ${ }^{1,5}$ \\ ${ }^{1}$ Center for Nanophotonics, FOM Institute AMOLF, c/o Philips Research Laboratories High Tech Campus 4, \\ AE Eindhoven 5656, The Netherlands \\ ${ }^{2}$ Optics Research Group, Delft University of Technology, CJ Delft 2628, The Netherlands \\ ${ }^{3}$ Center for Nano and Biophotonics, Ghent University, Ghent B-9000, Belgium \\ ${ }^{4}$ Physics and Chemistry of Nanostructures, Ghent University, Ghent B-9000, Belgium \\ ${ }^{5}$ COBRA Research Institute, Eindhoven University of Technology, P.O. Box 513, MB Eindhoven 5600, The Netherlands \\ ${ }^{*}$ Corresponding author: s.rodriguez@amolf.nl
}

Received December 10, 2012; accepted February 28, 2013;

posted March 7, 2013 (Doc. ID 181421); published April 3, 2013

\begin{abstract}
We numerically demonstrate that a periodic array of metallic nanorods sustains a maximum near-field enhancement and a far field (FF)-induced transparency at the same energy and in-plane momentum. The coupling of bright and dark plasmonic lattice resonances, and electromagnetic retardation along the nanorod length, are responsible for this effect. A standing wave with a quadrupolar field distribution is formed, giving rise to a collective suppression of FF scattering and simultaneously enhanced local fields. (c) 2013 Optical Society of America

OCIS codes: $240.6680,250.5403,050.2770,260.5740$.
\end{abstract}

Resonance phenomena are at the heart of nearly any approach toward controlling light. The enhancement of an optical system's response at resonance is normally associated with an increased light extinction. In the presence of surface electromagnetic waves, the resonant condition requires a careful reconsideration, since the spectrum of radiation may be different in the near field (NF) with respect to the far field (FF) [ $\underline{1}-\underline{4}]$. Thus, a frequency of maximum NF enhancement may not coincide with an extinction maximum. Such differences have a profound significance for the field of metallic nano-optics, where optical antennas are commonly designed to manipulate NFs, but characterized by their FF spectra [5].

In this Letter, we demonstrate through finite-difference in time-domain (FDTD) simulations that a diffractive array of plasmonic nanorods sustains a maximum NF enhancement and an FF-induced transparency at the same energy and in-plane momentum. This effect can be regarded as a collective counterpart of electromagnetically induced transparency (EIT) in plasmonic systems [6-8]. Therein, destructive interference between two localized modes induces a narrow transparency window within a broad resonance. One of the two modes, henceforth called bright, couples strongly to radiation and has a broad linewidth. The other mode, henceforth called dark, couples weakly to radiation and has a narrow linewidth. Dark modes have attracted interest for the realization of SPASERS [9], subwavelength guiding of optical radiation [10], enhanced spontaneous emission [11], and sensing $[12,13]$. In this work the bright and the dark modes are both collective resonances (their quality factors increase with the number of particles in the array [14]) rather than localized. A key point we address is how the NF resonance at the FF-induced transparency can be designed by varying the length of the nanorods.

All simulations are done with an in-house developed FDTD model [15]. The computational domain consists of a unit cell with Bloch-Floquet boundary conditions on the sides and perfectly matched layers on the top and bottom, wherein the incident wave propagates downward. We investigate arrays of gold nanorods in a rectangular lattice with constants $a_{x}=600 \mathrm{~nm}$ and $a_{y}=300 \mathrm{~nm}$. The surrounding medium is homogeneous with refractive index $n=1.46$ (silica). The dielectric function of gold is taken from Palik [16] and fitted with a Drude model. The incident light is polarized along the short axis of the nanorods. The transmittance (reflectance) is calculated by integrating the vertical timeaveraged Poynting vector on a plane below (above) the array. For the reflectance, the incident field is subtracted. As an indication of the NF intensity enhancement (NFIE), we compute $|E|^{2} /\left|E_{0}\right|^{2}$, with $E$ the total electric field and $E_{0}$ the incident field, at a plane intersecting the nanorods at their mid-height.

Figure 1 shows FF and NF spectra for an array of nanorods with dimensions $250 \mathrm{~nm} \times 110 \mathrm{~nm} \times 40 \mathrm{~nm}$. The zeroth-order transmittance $T_{0}$ in Fig. 1(a), reflectance $R_{0}$ in Fig. 1(b), NFIE in Fig. 1(d), and $1-R_{0}-T_{0}$ in Fig. 1(e), are shown in color as a function of the incident photon energy and the wave vector component parallel to the long axis of the nanorods $\vec{k}_{\|}=k_{0} \sin \left(\theta_{\text {in }}\right) \hat{x}$, with $k_{0}$ the magnitude of the free-space wave vector. The $(+1,0)$ and $(-1,0)$ Rayleigh anomalies (RAs) - diffraction orders radiating in the plane of the array-are indicated by the solid and dash-dot lines, respectively. Their dispersion is given by $E\left(k_{\|}\right)=(\hbar c / n)\left|k_{\|}+m G_{x}\right|$, where $m$ is the order of diffraction and $G_{x}=2 \pi / a_{x}$ is the $x$-component of the reciprocal lattice vector.

The two dips (peaks) in $T_{0}\left(R_{0}\right)$ redshifted with respect to the RAs and following their dispersion are hybrid photonic-plasmonic Fano resonances [17-20]. These

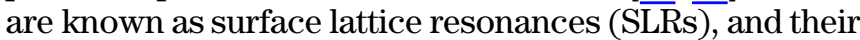
narrow linewidth stems from a collective suppression of radiative damping by the diffractive coupling of localized surface plasmons $[14,21]$. The SLR associated with the $(-1,0)$ order is bright, as its dispersion flattens and its extinction increases near normal incidence. In contrast, a narrowing linewidth and diminishing extinctionsignatures of subradiant damping-are observed for the $(+1,0)$ SLR as $k_{\|}$decreases and the mode becomes 
dark at normal incidence [22]. The mutual coupling of bright and dark SLRs leads to an anticrossing in their dispersion relation at $k_{\|}=0$, i.e., a frequency gap opens [22]. This anticrossing leads to a small gap near $1.37 \mathrm{eV}$ in Figs. 1(a) and 1(b). Much larger gaps were reported for identical lattices with different nanorod sizes in [22].

The NFIE features in Fig. 1(d) are very similar to those observed in $R_{0}$ and $T_{0}$ in the high $k_{\|}$regime, but a strong discrepancy arises near $k_{\|}=0.1 \mathrm{rad} / \mu \mathrm{m}$. Figure $\underline{1(\mathrm{e})}$ shows $1-R_{0}-T_{0}$, which for energies below the RAs is exactly the absorptance in the metal. As expected, there is a close correspondence between absorption and NFIE. In Fig. 1(c) we plot cuts of Figs. $\underline{1(a)}$ and 1(b) at $k_{\|}=0.1 \overline{\mathrm{rad}} / \mu \mathrm{m}$, and Fig. 1(f) shows cuts of Figs. 1 (d) and $1(\mathrm{e})$ at the same value of $k_{\|}$, which is indicated by the vertical dashed line in all dispersion diagrams. The dips (peaks) in $T_{0}\left(R_{0}\right)$ at 1.355 and $1.385 \mathrm{eV}$ in Fig. $1(\mathrm{c})$ correspond to SLRs associated with the $(-1,0)$ and $(+1,0)$ diffraction orders, respectively. A FF-induced transparency is observed as a dip (peak) in $R_{0}\left(T_{0}\right)$ between the two SLRs. In contrast, Fig. 1(f) displays a single peak in the NFIE and in absorptance at the same energy of the FF-induced transparency.
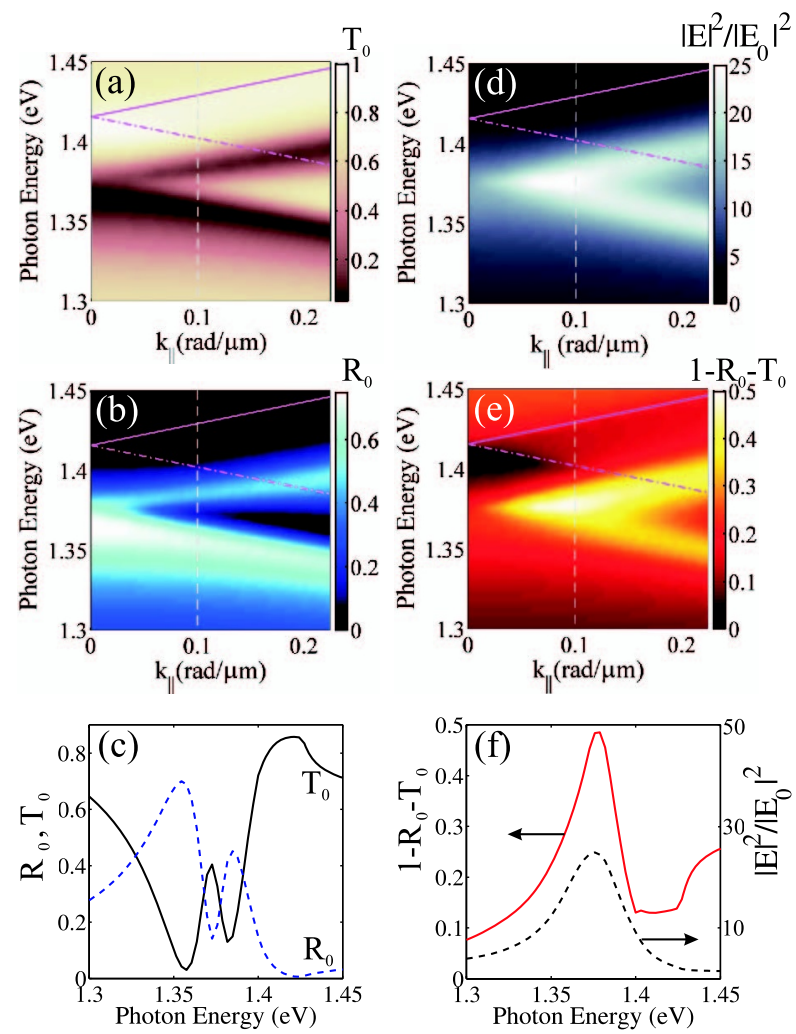

Fig. 1. Zeroth-order (a) transmittance $T_{0}$, (b) reflectance $R_{0}$, and (c) cut-of (a) and (b) at $k_{\|}=0.1 \mathrm{rad} / \mu \mathrm{m}$. (d) $1-R_{0}-T_{0}$, (e) average NFIE at a plane intersecting the nanorods at their mid-height, and (f) cut-of (d) and (e) as solid red line at $k_{\|}=0.1 \mathrm{rad} / \mu \mathrm{m}$. In (a), (b), (d), and (e), $k_{\|}=0.1 \mathrm{rad} / \mu \mathrm{m}$ is indicated by the vertical dashed lines; the solid and dash-dot lines indicate the $(+1,0)$ and $(-1,0)$ RAs. The nanorods have dimensions $250 \mathrm{~nm} \times 110 \mathrm{~nm} \times 40 \mathrm{~nm}$. Animations of the real, $y$ component of the electric field in the plane of the array are shown in Media 1 and Media 2 for the high- and low-energy extinction peaks in (c). Media 3 holds for the FF-induced transparency between these peaks, which is also at the peak in (f).
The contrast between the FF and NF spectrum of this nanorod array derives from the interference between the SLRs, and the associated retardation of the scattered field along the nanorod length. These processes are governed by the geometry of the nanorods, and in particular their length $L$. Figure 2 shows $T_{0}$ in (a) and NFIE in (b), as a function of $L$, for arrays of nanorods with dimensions $L \mathrm{~nm} \times 110 \mathrm{~nm} \times 40 \mathrm{~nm}$ illuminated by a plane wave with $k_{\|}=0.08 \mathrm{rad} / \mu \mathrm{m}$ The $110 \mathrm{~nm}$ width and $40 \mathrm{~nm}$ height of these nanorods are identical to those reported in [22], so direct comparison can be made for $L=450 \mathrm{~nm}$. The high- and low-energy features correspond to the $(+1,0)$ and $(-1,0)$ SLRs. Their energy and linewidth vary with $L$ due to retardation and radiative damping. Notice that for $L \gtrsim 250 \mathrm{~nm}$ the $(+1,0)$ SLR is bright, whereas the $(-1,0)$ SLR is dark, as reported in [22]. The SLR properties are interchanged for $L \lesssim 250 \mathrm{~nm}$, such that the flattening of the band occurs for the $(-1,0)$ SLR and subradiant damping onsets for the $(+1,0)$ SLR, as observed in Fig. 1. Particularly interesting is the regime $230 \mathrm{~nm} \lesssim L \lesssim 270 \mathrm{~nm}$, where $T_{0}$ displays an anticrossing characteristic of coupled modes, but the NFIE displays a crossing of the two modes. In this regime the structure has a resonant $\mathrm{NF}$ at an FF-induced transparency, as discussed for Fig. 1 .

The field profile at the photon energy and $k_{\|}$of the NF resonance at the FF-induced transparency is shown in Fig. 3. Figure 3(a) shows the total electric field enhancement $|E| /\left|E_{0}\right|$ in color and the scattered field as arrows. The four hot-spots near the corners of the nanorods and the scattered field show the quadrupolar character of the mode. The inclined incidence breaks the symmetry of the mode, which manifests as a stronger field enhancement on the right side of each nanorod. This broken symmetry leads to a nonvanishing dipole moment, which allows the excitation of this mode and a finite extinction. Figure $3(\mathrm{~b})$ shows the differences in phase between the scattered and incident fields, i.e., $\phi_{\text {sca }}-\phi_{\text {inc. }}$ Two values, which are $(-0.6 \pm 0.15) \pi$ (light blue) and ( $0.5 \pm$ $0.15) \pi$ (dark blue), prevail throughout space. Their difference, $1.1 \pi$, is close to the out-of-phase condition of $\pi$, thus resulting in a suppression of scattering. The phase distribution in Fig. 3(b) corresponds to the formation of a standing wave. The interference of two counterpropagating surface polaritons ( Media 1 and Media 2)

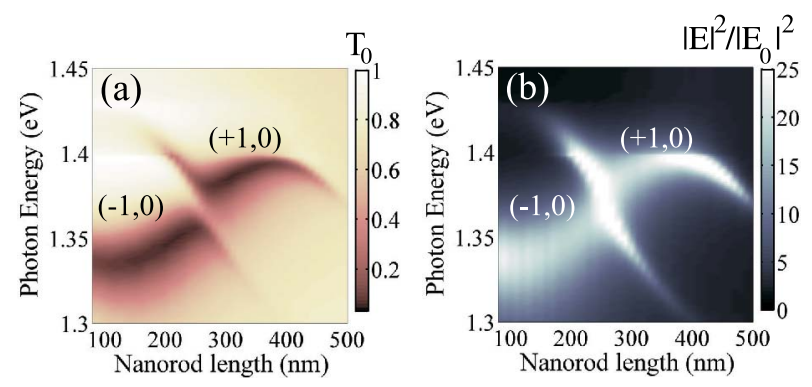

Fig. 2. (Color online) (a) Zeroth-order transmittance $T_{0}$ and (b) NFIE at a plane intersecting the nanorods at their mid-height. Arrays of nanorods with width $=110 \mathrm{~nm}$, height $=40 \mathrm{~nm}$, and variable length, are illuminated by a plane wave with $k_{\|}=0.08 \mathrm{rad} / \mu \mathrm{m}$. The labels near the SLRs indicate the associated diffraction order. 

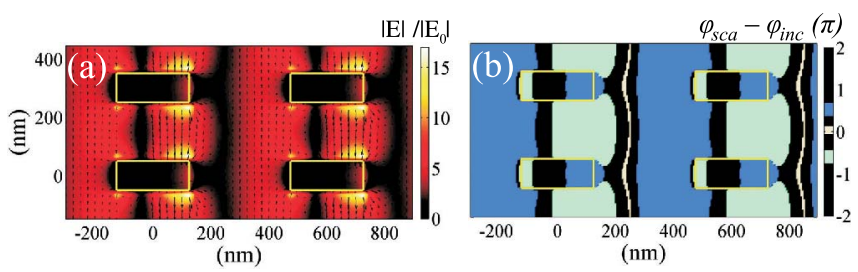

Fig. 3. (Color online) (a) Field enhancement (in color scale) and real part of the scattered field at an arbitrary phase (arrows) and (b) phase difference between the scattered and incident field in units of $\pi$. Both (a) and (b) are calculated at a plane intersecting the nanorods (delimited by the yellow lines) at their mid-height. The incident photon energy and in-plane wave vector correspond to the NF resonance at the FF-induced transparency.

creates the standing wave (Media 3, which is the timeevolution of the mode in Fig. 3).

In conclusion, a periodic array of plasmonic nanorods was shown to exhibit a resonant $\mathrm{NF}$ and FF-induced transparency at the same photon energy and in-plane momentum. We envisage this counterintuitive behavior to enable many of the key functionalities (e.g., enhanced light emission and sensing) pursued by plasmonic EIT analogs, but with the benefit of a collective resonance at the expense of tight field localization.

This work was supported by the Netherlands Foundation Fundamental Research on Matter (FOM) and the Netherlands Organization for Scientific Research (NWO), and is part of an industrial partnership program between Philips and FOM. This work is supported by NanoNextNL of the Government of the Netherlands and 130 partners. O. T. A. Janssen acknowledges the Dutch Technology Foundation STW, which is the applied science division of NWO, and the Technology Programme of the Ministry of Economic Affairs (project no. 10301). A. Omari acknowledges the Institute for the Promotion of Innovation through Science and Technology in Flanders (IWTVlaanderen). Z. Hens acknowledges BelSPo (IAP P7/35, photonics@be) and the FWO-Vlaanderen (project no. G.0794.10) for research funding.

\section{References}

1. A. V. Shchegrov, K. Joulain, R. Carminati, and J.-J. Greffet, Phys. Rev. Lett. 85, 1548 (2000).

2. G. W. Bryant, F. J. Garcia de Abajo, and J. Aizpurua, Nano Lett. 8, 631 (2008).

3. B. M. Ross and L. P. Lee, Opt. Lett. 34, 896 (2009).

4. B. Gallinet and O. J. F. Martin, Opt. Express 19, 22167 (2011).

5. P. Bharadwaj, B. Deutsch, and L. Novotny, Adv. Opt. Photon. 1, 438 (2009).

6. S. Zhang, D. A. Genov, Y. Wang, M. Liu, and X. Zhang, Phys. Rev. Lett. 101, 047401 (2008).

7. N. Liu, L. Langguth, T. Weiss, J. Kästel, M. Fleischhauer, T. Pfau, and H. Giessen, Nat. Mater. 8, 758 (2009).

8. P. Tassin, L. Zhang, T. Koschny, E. N. Economou, and C. M. Soukoulis, Phys. Rev. Lett. 102, 053901 (2009).

9. D. J. Bergman and M. I. Stockman, Phys. Rev. Lett. 90, 027402 (2003).

10. M. Liu, T.-W. Lee, S. K. Gray, P. Guyot-Sionnest, and M. Pelton, Phys. Rev. Lett. 102, 107401 (2009).

11. S. Rodriguez, S. Murai, M. Verschuuren, and J. Gómez Rivas, Phys. Rev. Lett. 109, 166803 (2012).

12. F. Hao, Y. Sonnefraud, P. V. Dorpe, S. A. Maier, N. J. Halas, and P. Nordlander, Nano Lett. 8, 3983 (2008).

13. F. Neubrech, A. Pucci, T. W. Cornelius, S. Karim, A. Garcia-Etxarri, and J. Aizpurua, Phys. Rev. Lett. 101, 157403 (2008).

14. S. Rodriguez, M. Schaafsma, A. Berrier, and J. Gómez Rivas, Physica B 407, 4081 (2012).

15. O. Janssen, "Rigorous simulations of emitting and nonemitting nano-optical structures," Ph.D. thesis (Delft University, 2010).

16. E. Palik, Handbook of Optical Constants of Solids (Academic, 1985).

17. S. Zou and G. C. Schatz, J. Chem. Phys. 121, 12606 (2004).

18. Y. Chu, E. Schonbrun, T. Yang, and K. B. Crozier, Appl. Phys. Lett. 93, 181108 (2008).

19. V. G. Kravets, F. Schedin, and A. N. Grigorenko, Phys. Rev. Lett. 101, 087403 (2008).

20. B. Auguié and W. L. Barnes, Phys. Rev. Lett. 101, 143902 (2008).

21. W. Zhou, Y. Hua, M. D. Huntington, and T. W. Odom, J. Phys. Chem. Lett. 3, 1381 (2012).

22. S. Rodriguez, A. Abass, B. Maes, O. Janssen, G. Vecchi, and J. Gómez Rivas, Phys. Rev. X 1, 021019 (2011). 